Gut and Liver, Vol. 13 No. 4, July 2019, pp. 450-460

\title{
The Use of Foxa2-Overexpressing Adipose Tissue-Derived Stem Cells in a Scaffold System Attenuates Acute Liver Injury
}

\author{
Yeon Ji Chae ${ }^{1}$, Dae Won Jun ${ }^{1,2}$, Jai Sun Lee ${ }^{1}$, Waqar Khalid Saeed ${ }^{2}$, Hyeon Tae Kang ${ }^{1}$, Kiseok Jang ${ }^{3}$, and Jin Ho Lee ${ }^{4}$ \\ ${ }^{1}$ Department of Translational Medicine, Hanyang University Graduate School of Biomedical Science and Engineering, Departments of ${ }^{2}$ Internal \\ Medicine and ${ }^{3}$ Pathology, Hanyang University College of Medicine, Seoul, and ${ }^{4}$ Department of Advanced Materials, Hannam University, \\ Daejeon, Korea
}

Background/Aims: For the clinical application of stem cell therapy, functional enhancement is needed to increase the survival rate and the engraftment rate. The purpose of this study was to investigate functional enhancement of the paracrine effect using stem cells and hepatocyte-like cells and to minimize stem cell homing by using a scaffold system in a liver disease model. Methods: A microporator was used to overexpress Foxa2 in adipose tissue-derived stem cells (ADSCS), which were cultured in a poly(lactic-co-glycolic acid) (PLGA) scaffold. Later, the ADSCs were cultured in hepatic differentiation medium for 2 weeks by a 3-step method. For in vivo experiments, Foxa2-overexpressing ADSCs were loaded in the scaffold, cultured in hepatic differentiation medium and later were implanted in the dorsa of nude mice subjected to acute liver injury (thioacetamide intraperitoneal injection). Results: Foxa2-overexpressing ADSCs showed greater increases in hepatocyte-specific gene markers (alpha fetoprotein [AFP], cytokeratin 18 [CK18], and albumin), cytoplasmic glycogen storage, and cytochrome P450 expression than cells that underwent the conventional differentiation method. In vivo experiments using the nude mouse model showed that 2 weeks after scaffold implantation, the mRNA expression of AFP, CK18, dipeptidyl peptidase 4 (CD26), and connexin 32 (CX32) was higher in the Foxa2-overexpressing ADSCs group than in the ADSCs group. The Foxa2-overexpressing ADSCs scaffold treatment group showed attenuated liver injury without stem cell homing in the thioacetamideinduced acute liver injury model. Conclusions: Foxa2-overexpressing ADSCs applied in a scaffold system enhanced hepatocyte-like differentiation and attenuated acute liver damage in an acute liver injury model without homing effects. (Gut Liver 2019;13:450-460)

Key Words: Liver failure, acute; Mesenchymal stem cell; Scaffold; Foxa2

\section{INTRODUCTION}

Adipose tissue-derived stem cells (ADSCs) are the most easily and abundantly acquired adult stem cells. ADSCs have differentiation abilities and stem cell characteristics similar to those of other adult stem cells, ${ }^{1}$ and using an individual's own ADSCs yields an important clinical advantage.

Many studies have demonstrated the beneficial effects of stem cell therapy in various liver diseases; however, stem cell therapy also has disadvantages, such as the low differentiation efficiency, short lifespan of stem cells, and the high cost of mass production. $^{2}$ To overcome these limitations and to enhance the efficacy of stem cell therapy, various studies have been conducted with different techniques.

Methods involving hypoxic pretreatment states, gene transfer and pretreatment with cytokines have been reported. ${ }^{2-5}$ Some studies on liver diseases have used hepatocyte nuclear factor $4 \alpha$ (HNF4 $\alpha$ ), forkhead box protein (Foxa2), hepatocyte growth factor (HGF) and insulin-like growth factor I gene transfer in association with stem cell therapy. ${ }^{6-10}$ Foxa2 activates hepatocytespecific genes such as albumin (ALB) and transthyretin and is also known as a major transcription factor for liver differentiation. ${ }^{11,12}$ Foxa2 overexpression in bone marrow-derived stem cells has been associated with significant increases in hepatocyte

Correspondence to: Dae Won Jun ${ }^{\mathrm{a}}$ and Kiseok Jang ${ }^{\mathrm{b}}$

${ }^{a}$ Department of Internal Medicine, Hanyang University College of Medicine, 222-1 Wangsimni-ro, Seongdong-gu, Seoul 04763, Korea

Tel: +82-2-2290-8338, Fax: +82-2-972-0068, E-mail: noshin@hanyang.ac.kr

${ }^{\mathrm{b}}$ Department of Pathology, Hanyang University College of Medicine, 222-1 Wangsimni-ro, Seongdong-gu, Seoul 04763, Korea

Tel: +82-2-2290-8248, Fax: +82-2-972-0068, E-mail: medartisan@hanyang.ac.kr

Received on May 23, 2018. Revised on September 15, 2018. Accepted on October 17, 2018. Published online March 15, 2019

pISSN 1976-2283 eISSN 2005-1212 https://doi.org/10.5009/gnl18235

Yeon Ji Chae and Jai Sun Lee contributed equally to this work as first authors.

@ This is an Open Access article distributed under the terms of the Creative Commons Attribution Non-Commercial License (http://creativecommons.org/licenses/by-nc/4.0) which permits unrestricted non-commercial use, distribution, and reproduction in any medium, provided the original work is properly cited. 
functional markers such as ALB, Cytochrome P450, and alpha-1 antitrypsin (AAT). ${ }^{13}$ Moreover, Foxa2-overexpressing rat bone marrow-derived mesenchymal stem cell (rBM-MSC) therapies are more effective in treating hepatic fibrosis than conventional stem cell therapies. ${ }^{7}$ However, safety issues such as off-target migration of gene-transferred cells have not been resolved in in vivo models. ${ }^{14}$ Moreover, there is a risk of thrombus formation when stem cells are administered intravascularly because of different stem cell sizes. ${ }^{15}$ Therefore, to minimize these risks, treatment options without stem cell homing have been considered.

Recent studies have reported that stem cell-secreted cytokines play a more positive role in tissue recovery and regeneration than stem cell transfer to tissues and differentiation into healthy cells. ${ }^{16,17}$ The use of stem cell paracrine effects may be an approach to improve the stability of stem cell therapies by avoiding direct stem cell injection into tissues.

In this study, we investigated whether the use of partially differentiated ADSCs overexpressing Foxa2 in a scaffold system could help maximize the efficacy of ADSCs. We further investigated the additional benefits of using partially differentiated hepatocyte-like cells under a scaffold system in an acute liver injury model.

\section{MATERIALS AND METHODS}

\section{Cell isolation and culture}

Human ADSCs (PT-5006, Lonza, Walkersville, MD, USA) were purchased from Lonza. Cells from the third passage were used; the cell pellet was resuspended in complete Dulbecco's modified Eagle's medium with F12 (DMEM F12) containing 10\% fetal bovine serum and 1\% penicillin-streptomycin and plated on a 6-mm dish at $37^{\circ} \mathrm{C}$ in an atmosphere of $5 \% \mathrm{CO}_{2}$.

\section{Plasmids and molecular cloning}

The coding regions of Foxa2, HNF4 $\alpha$, and GATA4 (NM_ 021784.4, NM_000457.4, and NM_001308093.1) were amplified using a cDNA ORF clone (OriGene, Rockville, MD, USA) and cloned into a pEGFP-C1 vector (Clontech, Palo Alto, CA, USA). The pEGFP-C1 vector is a green fluorescent protein (GFP)-tagged plasmid and was used alone as a control (pEGFP-C1 group). This plasmid does not contain any foreign genes.

\section{Transfection using electroporation}

ADSCs were resuspended in resuspension buffer R (Invitrogen, Waltham, MA, USA) at a density of $1 \times 10^{6}$ cells $/ \mathrm{mL}$ and mixed with $1 \mu \mathrm{g}$ of the constructed plasmids for each gene. Electroporation was performed using a Microporator (Invitrogen) at 1,200 $\mathrm{V}$ in two pulses of $20 \mathrm{~ms}$ each. After electroporation, the cells were plated on a 6 -well plate at $37^{\circ} \mathrm{C}$ in an atmosphere of $5 \%$ $\mathrm{CO}_{2}$. The green fluorescence of EGFP-C1 was visualized using a Leica DMI 14000B inverted microscope (Leica Microsystems, Wetzlar, Germany) and confirmed by qRT-PCR (Supplementary
Fig. 1).

\section{ADSC seeding into PLGA scaffolds}

Poly(lactic-co-glycolic acid) (PLGA) scaffolds (MW: 115 kDa, Sigma-Aldrich; Pluronic F127) (professor J.H.L: pore size, 100 to $200 \mu \mathrm{m} ; \Phi, 7 \mathrm{~mm}$; T, $2 \mathrm{~mm}$ ) were loaded with $1 \times 10^{5}$ transfected ADSCs using a volume of $20 \mu \mathrm{L}$ on each side. To settle the ADSCs, the ADSC-loaded scaffolds were incubated for 2 hours in complete medium. After 24 hours, the transfection efficiency was determined using fluorescence microscopy. Later, differentiation was carried out in differentiation medium.

\section{Hepatocyte-like cell differentiation methods}

Three differentiation media were used for ADSC differentiation into hepatocyte-like cells. For the first (differentiation medium \# 1), $20 \mathrm{ng} / \mathrm{mL}$ epidermal growth factor (EGF) and $10 \mathrm{ng} /$ $\mathrm{mL}$ basic fibroblast growth factor (bFGF) were added to Iscove's modified Dulbecco's medium (IMDM), and differentiation was carried out for 2 days. In the second (differentiation medium \#2), IMDM was supplemented with a mixture of $20 \mathrm{ng} / \mathrm{mL} \mathrm{HGF}$, $10 \mathrm{ng} / \mathrm{mL}$ bFGF and $0.61 \mathrm{~g} / \mathrm{L}$ nicotinamide, and differentiation was carried out for 1 week. For the third (differentiation medium \#3), $20 \mathrm{ng} / \mathrm{mL}$ oncostatin M, $1 \mu \mathrm{mol} / \mathrm{L}$ dexamethasone and $50 \mathrm{mg} / \mathrm{mL}$ ITS premix were added to IMDM, and differentiation was carried out for 1 week.

\section{RNA isolation, real-time quantitative polymerase chain reaction (qRT-PCR) and reverse transcription PCR (RT- PCR)}

RNA was extracted from ADSCs and ADSCs/scaffolds using TRIzol reagent (Invitrogen), and cDNA was synthesized using a PrimeScript RT Reagent Kit (Takara Bio, Kusatsu, Japan). The scaffold was frozen in liquid nitrogen and crushed before TRIzol reagent was used. qRT-PCR was performed using a LightCycler 480 system (Roche Diagnostics, Mannheim, Germany) with LightCycler 480 SYBR Green I Master Mix (Roche Diagnostics). All samples were subjected to three repeated tests for accuracy. The primers for human Foxa2, HNF4 $\alpha$, GATA4, ALB, cytokeratin 18 (CK18), and alpha fetoprotein (AFP) and AFP and for mouse tumor necrosis factor- $\alpha$ (TNF- $\alpha$ ), interleukin (IL)-1 $\beta$, monocyte chemotactic protein-1 (MCP1) and IL-6 are shown in Supplementary Tables 1 and 2. For RT-PCR, RNA was extracted from each liver tissue sample using TRIzol reagent (Invitrogen) and cDNA was synthesized with a cDNA synthesis kit (iNtRON Biotechnology, Seongnam, Korea). The RT-PCR primers included those for human $\beta 2$-microglobulin (forward: 5'-GTGTCTGGGTTTCATCCATC, reverse: 5'-AATG CGGCATCTTCAACCTC) and mouse $\beta 2$-microglobulin (forward: 5'-TCAGTAACACAGTTCCACCC, reverse: 5'-GTTCAAAT GAATCTTCAGAGCAT). 


\section{Immunofluorescence}

The scaffold paraffin block was sectioned, and immunofluorescence was performed to determine cytochrome P450 expression. After deparaffinization using Histo-Clear, permeabilization was performed with $0.1 \%$ Triton X-100 in phosphate-buffered saline, and the sections were blocked with 10\% normal goat serum and 1\% bovine serum albumin. Anti-cytochrome P450 2E1 (ab28146; Abcam, Cambridge, UK) was used, and goat antirabbit IgG H\&L (Alexa Fluor 488) (ab150077; Abcam) was used as the secondary antibody. To identify human-specific markers, anti-mitochondrial antibody, surface of intact mitochondria, clone 113-1 (MAB1273; Merck Millipore, Darmstadt, Germany) was used, and goat anti-mouse IgG (Alexa Fluor 488) (ab150113; Abcam) was used as the secondary antibody. Immunofluorescence quantification was performed with ImageJ software (National Institutes of Health, Bethesda, MD, USA).

\section{Animals and experimental procedures}

C57BL/6 and nude mice (6 weeks old) were purchased from Orient Animal Laboratory, Seoul, South Korea. Nude mice were transplanted with ADSCs/scaffolds in their dorsal subcutaneous tissue for in vivo cultures (HY-IACUC-16-0015). A liver injury model was established using C57BL/6 mice to determine the effects of the transfected ADSCs/scaffolds. All C57BL/6 mice received thioacetamide (TAA; $200 \mathrm{mg} / \mathrm{kg}$, intraperitoneal) (Sigma-Aldrich, 163678) for the first 2 days, and the scaffold was implanted in the dorsal subcutaneous tissue of the mice immediately after the first injection. ADSCs/scaffolds and Foxa2transfected ADSCs/scaffolds were incubated in differentiation medium \# 1 for 1 day before being implanted. The TAA-only group (group 1) was injected with TAA once daily for 2 consecutive days, and scaffolds with no cells were implanted. Group 2 was implanted with pEGFP-C1-transfected ADSCs/scaffolds, while group 3 was implanted with Foxa2-transfected ADSCs/ scaffolds. The mice in all groups were given dorsal incisions to minimize surgical variations. After a week, the surviving mice were anesthetized, liver and blood samples were obtained, and the scaffolds were retrieved (HY-IACUC-16-0034). All the experimental procedures were approved by the Hanyang Institutional Animal Care and Use Committee.

\section{Histological analysis}

The liver tissue and implanted scaffolds were fixed in $4 \%$ paraformaldehyde. The paraffin blocks were prepared by fixation, washing, dehydration, clearing, paraffin infiltration and embedding. Liver tissue and the retrieved scaffolds were sectioned (4- and 8- $\mu \mathrm{m}$-thick sections, respectively) and processed for further experiments. Hematoxylin and eosin (H\&E) staining was performed, and the necrotic area was evaluated by a single blinded pathologist. For liver function evaluation, periodic acidSchiff staining (PAS Staining Kit, 1.01646.0001; Merck Milli- pore) was performed to analyze glycogen storage capacity. PAS staining was quantified by the immunoreactive score (IRS). The intensity of glucose staining was scored as follows: no reaction received a score of 0 , a weak color reaction received a score of 1 , a moderate-intensity color reaction received a score of 2 , and an intense reaction received a score of 3 . In addition, the extent of glucose staining (\%) was scored as follows: a lack of positive cells denoted a score of $0,<25 \%$ positive cells denoted a score of $1,25 \%$ to $50 \%$ positive cells denoted a score of $2,51 \%$ to $75 \%$ positive cells denoted a score of 3 , and $>75 \%$ positive cells denoted a score of 4 . To obtain the final score, the intensity score was multiplied by the extent score.

\section{Magnetic Luminex assay}

HGF, EGF, bFGF, vascular endothelial growth factor-A (VEGF-A), IL-10, IL-4, IL-13, TNF- $\alpha$, MCP-1, IL-1 $\beta$, and IL-6 secretion were assessed using a magnetic Luminex assay kit (R\&D Systems, Minneapolis, MN, USA). The cytokines secreted by Foxa2-overexpressing ADSCs were assessed using culture medium collected after 2, 5, or 7 days of differentiation. Differentiation media \# 1 and \#2 were used as controls.

\section{Statistical analysis}

All experiments were independently repeated three times. The values are expressed as the mean \pm standard deviation. Statistical analysis was performed using SPSS for Windows version 18.0 (SPSS Inc., Chicago, IL, USA). One-way analysis of variance was performed to compare means, and a p-value $<0.05$ was considered significant.

\section{RESULTS}

\section{Hepatocyte differentiation using hepatic gene overex- pression in 2D conditions}

The mRNA expression of the hepatic differentiation markers AFP, ALB and CK18 was evaluated after the Foxa2, HNF4 $\alpha$, and GATA4 genes were overexpressed in ADSCs differentiated using conventional differentiation medium (Fig. 1A). Foxa2 overexpression increased the expression of AFP (18-fold increase), ALB (10-fold increase) and CK18 (16-fold increase) compared with normal culture conditions. Moreover, HNF4 $\alpha$ overexpression also increased the expression of AFP (3-fold increase), ALB (5-fold increase) and CK18 (3-fold increase) compared with normal differentiation conditions. The GATA4-overexpressing ADSC group also showed significantly higher expression of AFP (107-fold increase) and CK18 (50-fold increase) than the nonoverexpressing group. However, GATA4-overexpressing ADSCs did not show good viability. PAS staining revealed increased glycogen storage ability in the transfected cells compared to the control cells (Fig. 1B).

We therefore selected the Foxa2-overexpressing ADSC group for subsequent experiments (three-dimensional [3D] culture and 

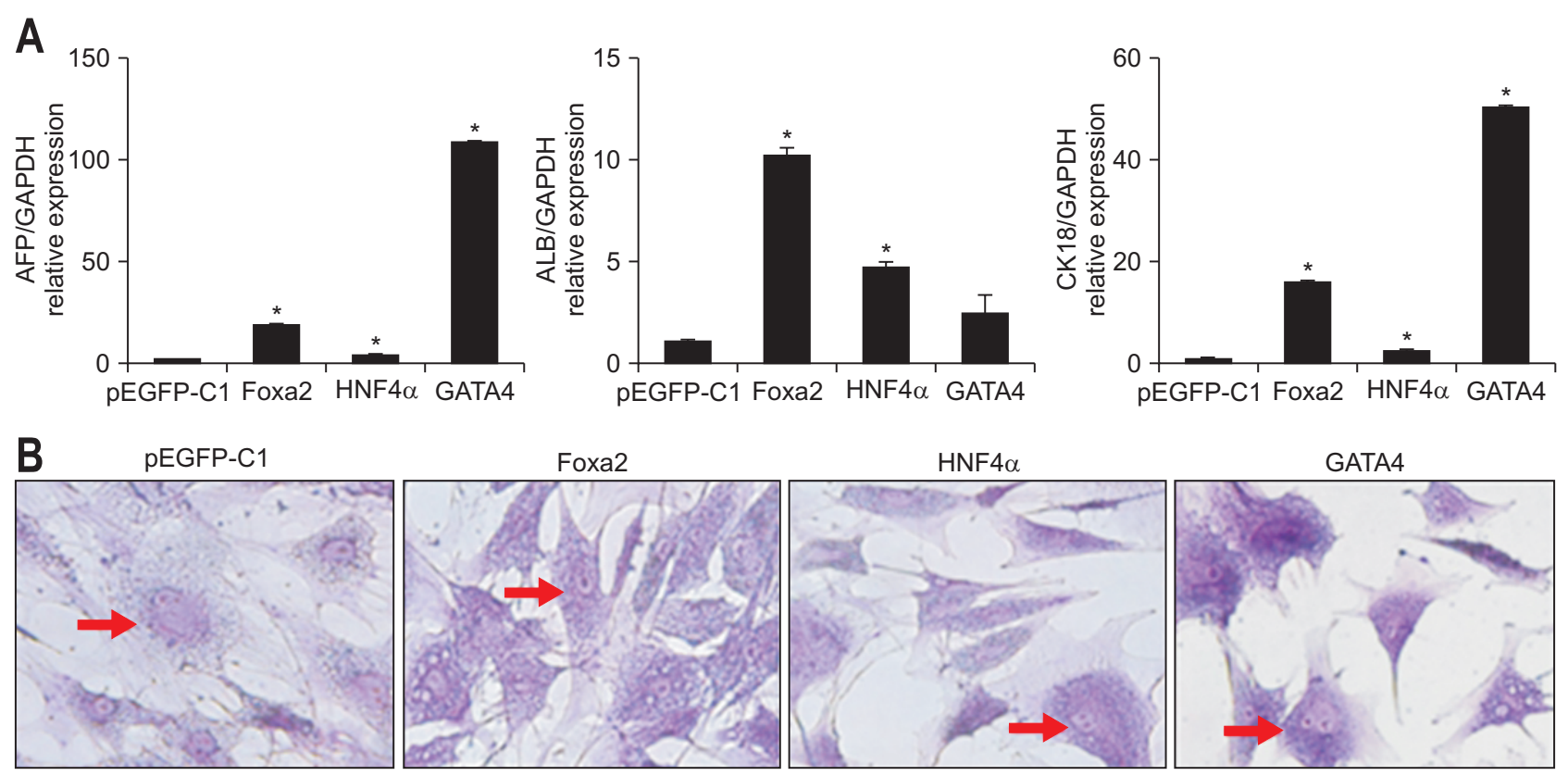

Fig. 1. Hepatic differentiation ability in two-dimensional (2D) culture. (A) Gene expression of hepatic differentiation markers (AFP, ALB, and CK18) in transfected ADSCs after 2 weeks measured by qRT-PCR. (B) Amount of cytoplasmic glycogen after the overexpression of various gene in 2D culture measured by periodic acid-Schiff (PAS) staining (PAS staining: blue, nuclei; purple, glycogen in cytoplasm; magnification: $\times 400$ ). The arrows indicate cytosol.

AFP, alpha fetoprotein, ALB, albumin; CK18, cytokeratin 18; ADSCs, adipose tissue-derived stem cells; qRT-PCR, real-time quantitative polymerase chain reaction; Foxa2, forkhead box protein. ${ }^{*} \mathrm{p}<0.05$.

in vivo culture) due to the successful induction of AFP, ALB and CK18 expression in two-dimensional (2D) cultures.

\section{Hepatocyte differentiation using Foxa2 overexpression in a 3D scaffold system (Foxa2-overexpressing ADSCs/ scaffolds)}

Next, we analyzed hepatic differentiation ability after overexpressing Foxa2 under conventional differentiation conditions in 3D cultures with PLGA scaffolds (Fig. 2). The mRNA levels of AFP, CK18, CD26 and CX32 were significantly higher in the Foxa2-overexpressing ADSC/scaffold group than in the conventional differentiation group (AFP: 4-fold higher, $p=0.01$; CK18: 2-fold higher, $p=0.028$; CD26: 3-fold higher, $p=0.024$; CX32: 28-fold higher, $\mathrm{p}<0.001$ ) (Fig. 2A). H\&E staining confirmed the presence of cells in the PLGA scaffold. Next, to evaluate hepatocyte function in Foxa2-overexpressing ADSCs, glycogen storage ability and cytochrome P450 expression were determined. The intensity of PAS staining was 1.7 -fold higher $(p=0.05)$ in the Foxa2-overexpressing ADSC/scaffold group than in the control group. However, the extent of PAS staining was not significantly different between the two groups. The final score obtained by multiplying the two scores (the IRS) was 1.8 -fold higher $(\mathrm{p}=0.05)$ in the Foxa2-overexpressing ADSC/scaffold group than in the control group. In 3D culture, cytochrome $\mathrm{P} 450$ expression was 2.2-fold higher $(\mathrm{p}<0.01)$ in the Foxa2-overexpressing ADSC/ scaffold group than in the control group (Fig. 2B and C).

Thus, Foxa2 overexpression promoted hepatic differentiation in an in vitro system. Next, we evaluated whether transfection of ADSCs with Foxa2 also promoted in vivo hepatic differentiation in a nude mouse model.

\section{Hepatic differentiation using Foxa2 overexpression and a PLGA scaffold system in an in vivo model}

We next investigated whether Foxa2 overexpression in $\mathrm{AD}$ SCs promotes differentiation into hepatocyte-like cells in vivo. Foxa2-overexpressing ADSCs and conventional ADSCs were injected into PLGA scaffolds and incubated in differentiation medium \#1 for hepatic differentiation. The next day, the PLGA scaffolds were implanted into mouse subcutaneous tissue (Fig. 3A). Blood vessel formation was observed around scaffolds and differentiated stem cells were observed through a scanning electron microscope (SEM) in both groups on day 14 after scaffold implantation (Fig. 3A and B).

The Foxa2-overexpressing ADSC/scaffold group had 6-fold higher AFP levels $(p<0.001), 5$-fold higher CK18 levels $(p=0.02)$, 2.5-fold higher CD26 levels ( $\mathrm{p}=0.001$ ), and 2.8-fold higher CX32 levels $(p<0.001)$ than the group of ADSCs without gene overexpression (Fig. 3C). After 2 weeks, the cells in the PLGA scaffolds implanted in the subcutaneous tissues of nude mice were identified by SEM and H\&E staining. To evaluate hepatocyte function, glycogen storage ability and cytochrome P450 expression were determined. The intensity of PAS staining was 1.7fold higher $(\mathrm{p}=0.05)$ in animals with the Foxa2-overexpressing $\mathrm{ADSC} /$ scaffold system than in control animals. In addition, the 
A
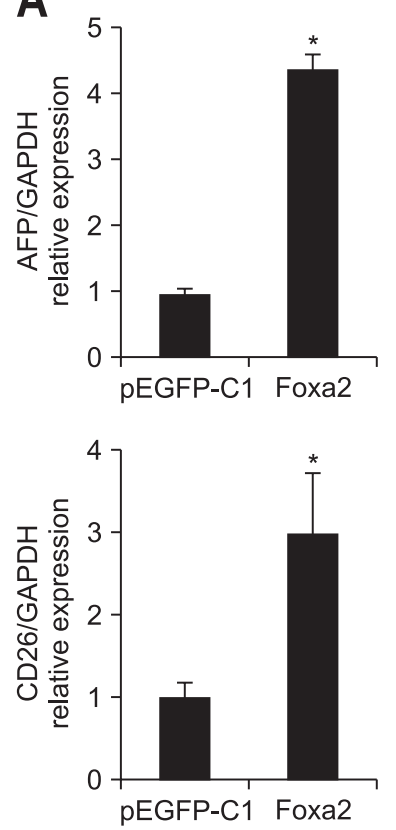
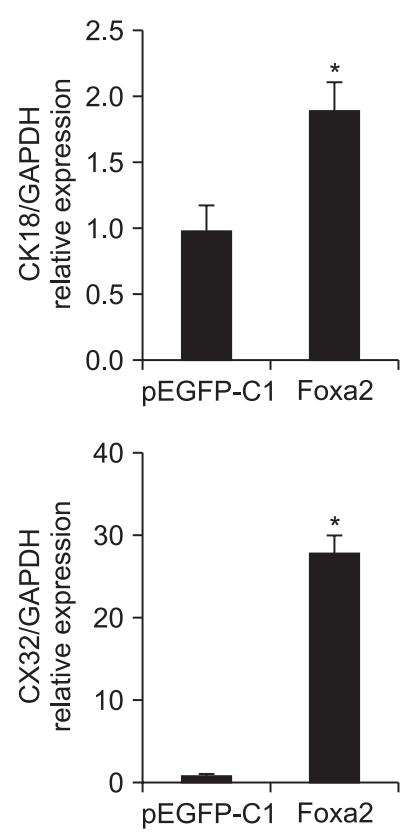
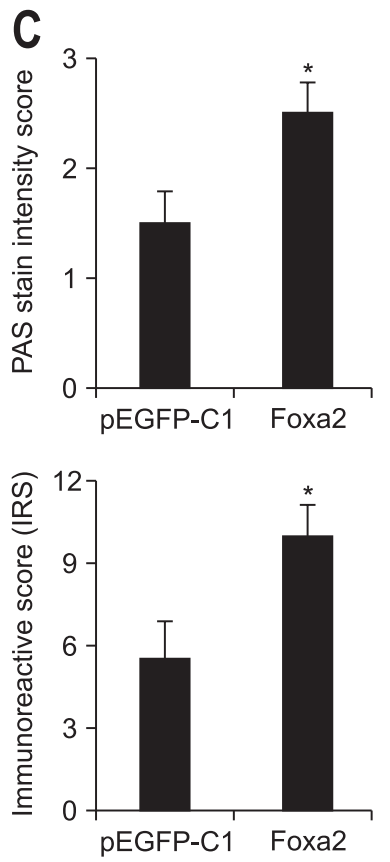
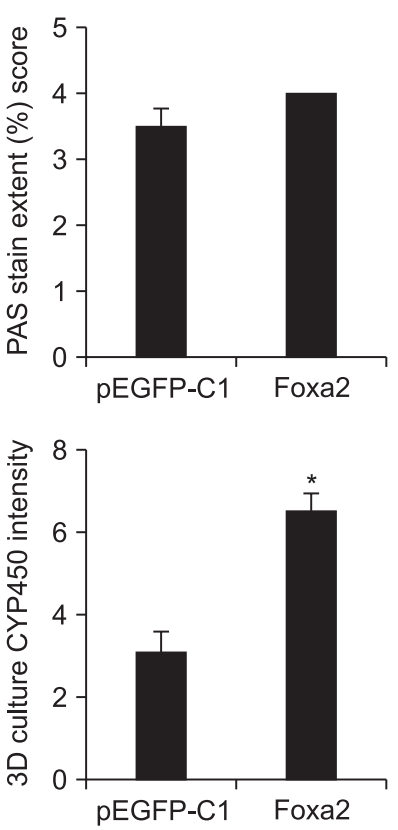

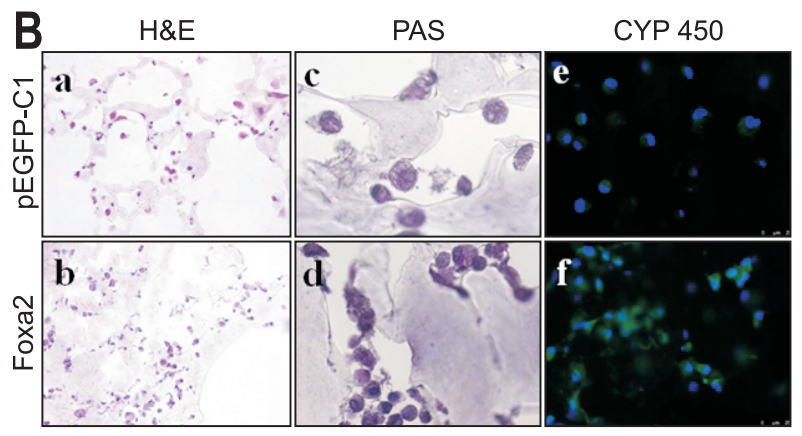

Fig. 2. Comparison of hepatic differentiation ability in three-dimensional (3D) culture using a scaffold. (A) qRT-PCR expression of hepatic differentiation markers (AFP, CK18, CD26, and CX32) in Foxa2-transfected ADSCs after 2 weeks in 3D culture. (B) H\&E staining, periodic acidSchiff (PAS) and cytochrome P450 immunofluorescence in Foxa2-overexpressing ADSCs in 3D culture (a and b: H\&E staining, blue - nuclei, pink - cytosol, ×400; c and d: PAS staining, blue - nuclei, purple - glycogen in cytoplasm, ×1,000; e and f: blue - nuclei, green - cytochrome 450 in cytosol, $\times 1,000)$. (C) Quantification of cytochrome P450 intensity and glucose expression.

qRT-PCR, real-time quantitative polymerase chain reaction; AFP, alpha fetoprotein; CK18, cytokeratin 18; CD26, dipeptidyl peptidase 4; CX32, connexin 32; ADSCs, adipose tissue-derived stem cells; Foxa2, forkhead box protein. * $\mathrm{p}<0.05$.

IRS was also 1.5-fold higher ( $\mathrm{p}=0.05$ ) in the Foxa2-overexpressing $\mathrm{ADSC} / \mathrm{scaffold}$ group than in the control group in vivo. Cytochrome P450 expression was 4.2 -fold higher $(\mathrm{p}<0.01)$ in the Foxa2-overexpressing ADSC/scaffold group than in the control model group (Fig. 3D and E).

Thus, Foxa2 overexpression promoted hepatic differentiation in an in vivo system. Next, we evaluated whether transfection of ADSCs with Foxa2 attenuated acute liver damage in an acute liver injury model.

\section{Foxa2 overexpression in ADSCs in a PLGA scaffold sys- tem attenuated liver injury in acute liver injury model}

An acute liver injury model was induced by intraperitoneal injection of TAA for 2 consecutive days. On day 1 of TAA injection, Foxa2-overexpressing ADSCs/scaffolds and non- overexpressing ADSCs/scaffolds were inserted into dorsal subcutaneous tissues of nude mice. Liver tissues and scaffolds were retrieved after a week. Inflammatory cells and necrotic areas were observed near the portal triad following TAA injection, but the necrotic area was significantly lower in the Foxa2-overexpressing ADSC/scaffold group than in the non-overexpressing group (Fig. 4A). Later, liver function tests were performed on blood samples. ALB synthesis was significantly increased and total bilirubin was significantly decreased in the Foxa2-overexpressing ADSC/scaffold group compared to non-overexpressing group. However, aspartate aminotransferase (AST) and alanine aminotransferase (ALT) levels did not differ between the ADSC/ scaffold group and the Foxa2-overexpressing ADSC/scaffold group (Fig. 4B). Liver tissue MCP1 mRNA expression was significantly lower $(\mathrm{p}=0.034)$ in the Foxa2-overexpressing group 
A

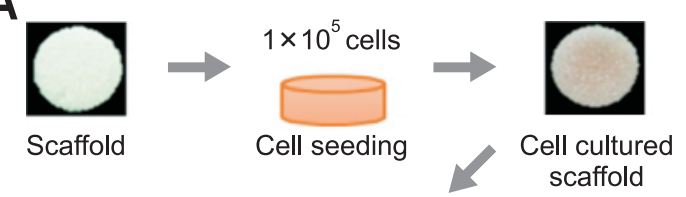

Scaffold implant into mouse
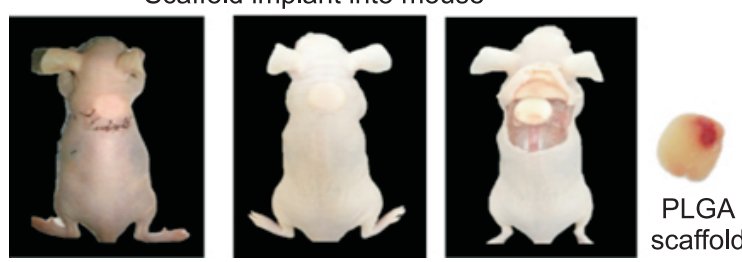

B

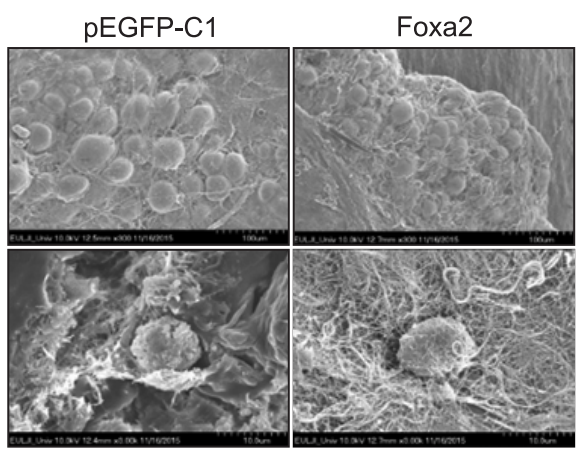

C
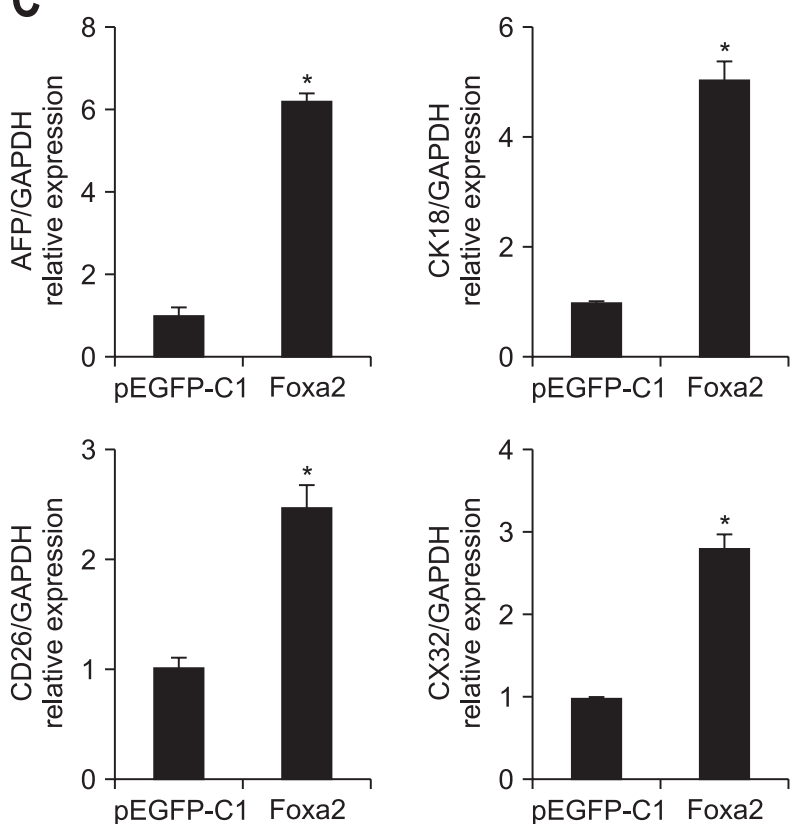

D
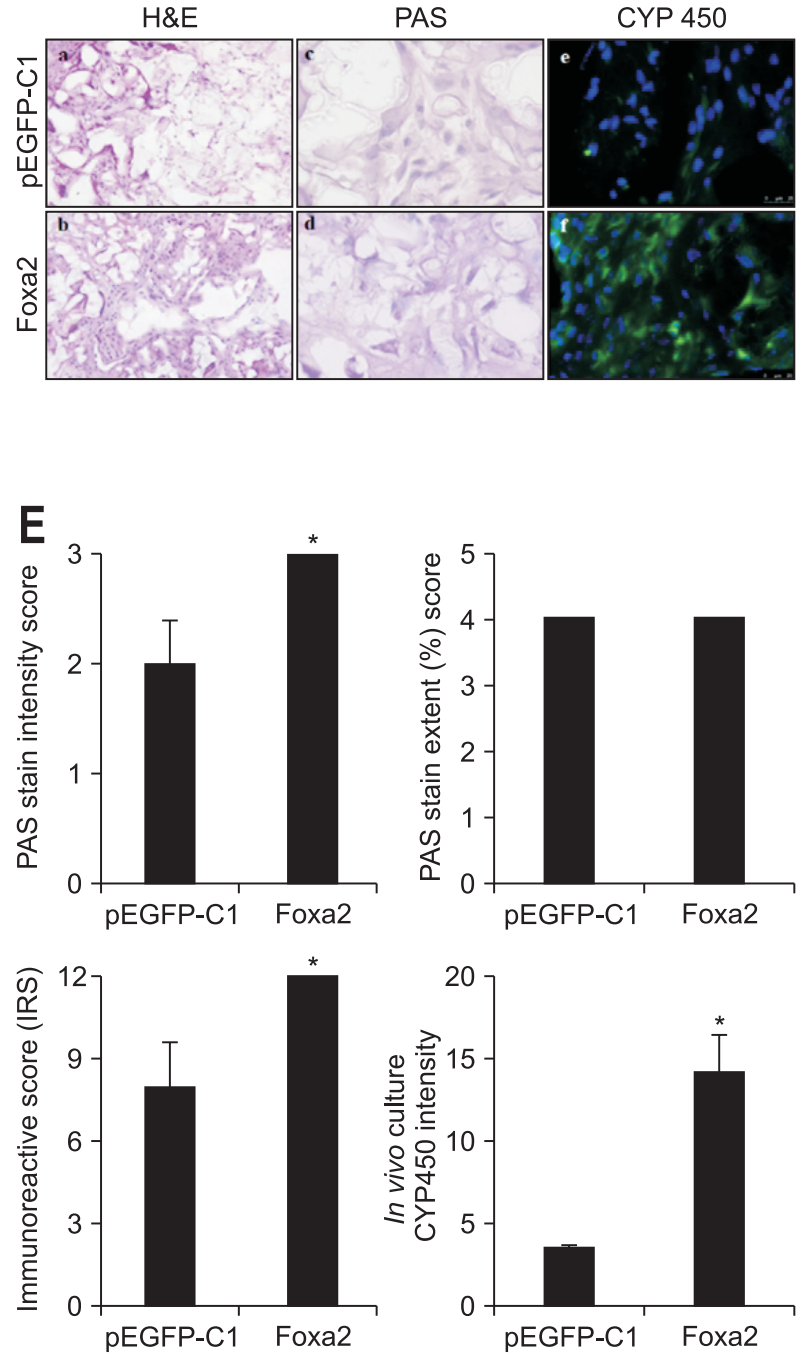

Fig. 3. Comparison of hepatocyte differentiation in a scaffold during in vivo culture. (A) In vivo culture design of scaffold preparation and implantation into nude mice. (B) Scanning electron microscopy of ADSCs in the PLGA scaffold. (C) qRT-PCR expression of hepatic differentiation markers (AFP, CK18, CD26 and CX32) in Foxa2-transfected ADSCs after 2 weeks in nude mice. (D) H\&E staining, periodic acid-Schiff (PAS) staining and immunofluorescent staining for cytochrome P450 activity after Foxa2 gene overexpression in in vivo culture (a and b: H\&E staining, blue - nuclei, pink - cytosol, $\times 400$; c and d: PAS staining, blue - nuclei, purple - glycogen in cytoplasm, $\times 1,000$; e and f: blue - nuclei, green cytochrome 450 in cytosol, $\times 1,000$ ). (E) Quantification of cytochrome P450 intensity and glucose expression.

ADSCs, adipose tissue-derived stem cells; PLGA, poly(lactic-co-glycolic acid); qRT-PCR, real-time quantitative polymerase chain reaction; AFP, alpha fetoprotein; CK18, cytokeratin 18; CD26, dipeptidyl peptidase 4; CX32, connexin 32; Foxa2, forkhead box protein. * ${ }^{*}<0.05$. 
A
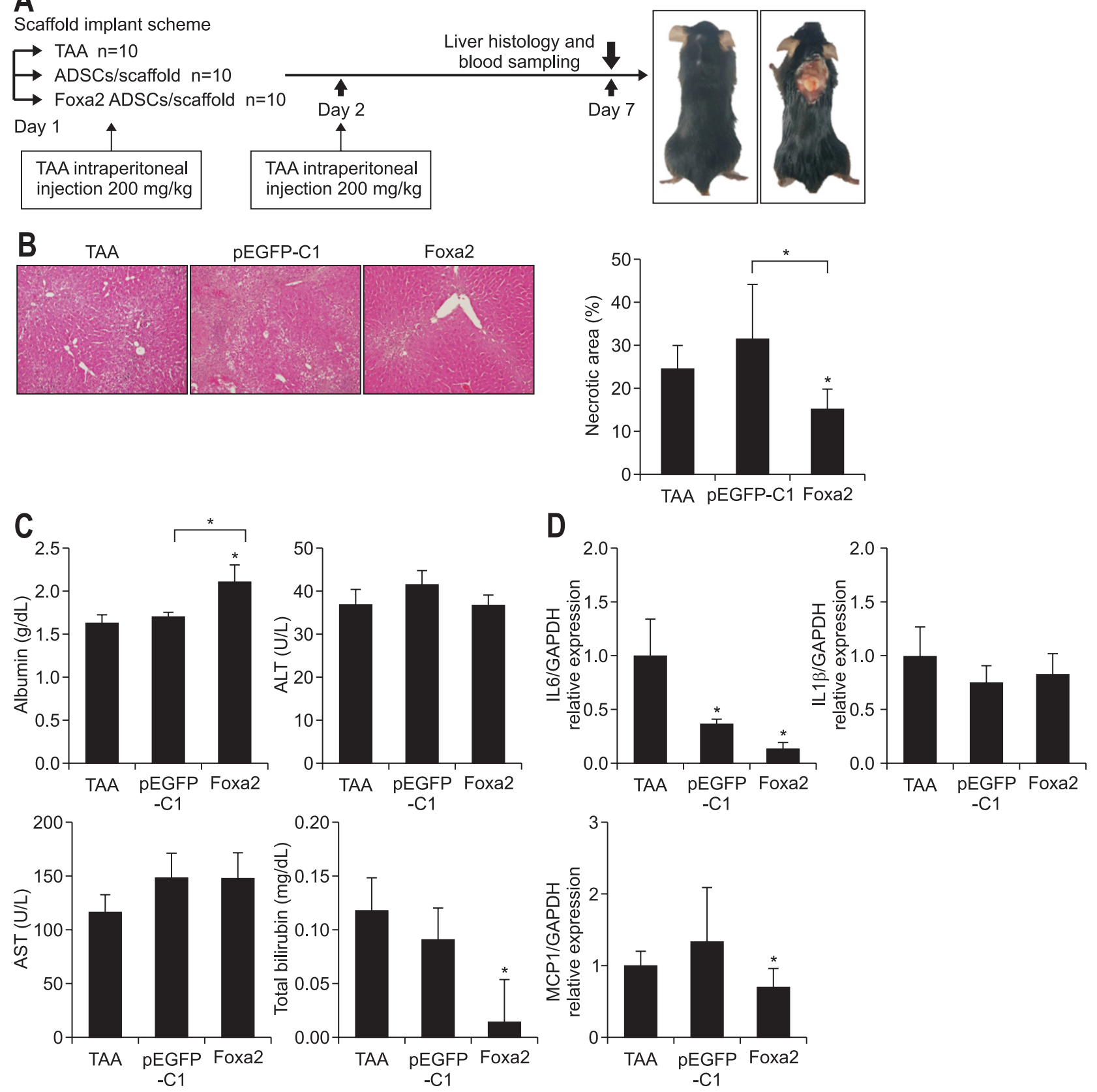

Fig. 4. The Foxa2-overexpressing ADSCs scaffold treatment group showed attenuated liver injury in a thioacetamide-induced acute liver failure model. Hematoxylin and eosin staining and necrotic area percentage. (A) The scheme of experimental design. (B) H\&E staining and necrotic area percentage. (C) Expression of several hepatic enzymes (albumin, ALT, AST and total bilirubin) in serum. (D) The gene expression of inflammation markers (IL-1 $\beta$ and MCP1) was compared among the thioacetamide (TAA) injection group, ADSCs/scaffold implanted group and Foxa2-overexpressing ADSCs/scaffold implanted group in mouse livers.

Foxa2, forkhead box protein; ADSCs, adipose tissue-derived stem cells; ALT, alanine aminotransferase; AST, aspartate aminotransferase; IL, interleukin; MCP1, monocyte chemoattractant protein-1. ${ }^{*} \mathrm{p}<0.05$.

than in the TAA-only group (Fig. 4C).

\section{Stem cell homing and changes in the scaffolds}

Stem cell migration into the injured liver in the ADSC/scaffold-implanted groups was evaluated using PCR and immunofluorescence (Fig. 5). Based on human and mouse $\beta 2$-globulin housekeeping gene expression and human-specific immuno- fluorescence staining, no stem cell migration into damaged liver tissue was observed (Fig. 5A and B).

Immunofluorescence staining using a human-specific antibody confirmed that most of the cells in the scaffolds were human ADSCs. PAS staining was used to analyze the differentiation of injected ADSCs into hepatocyte-like cells, and the results showed higher glycogen storage ability in the Foxa2-overex- 
A

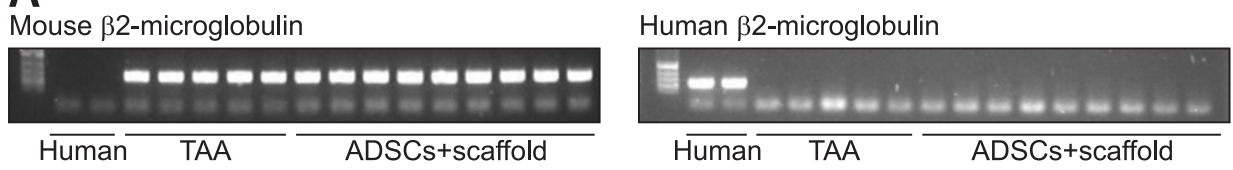

B

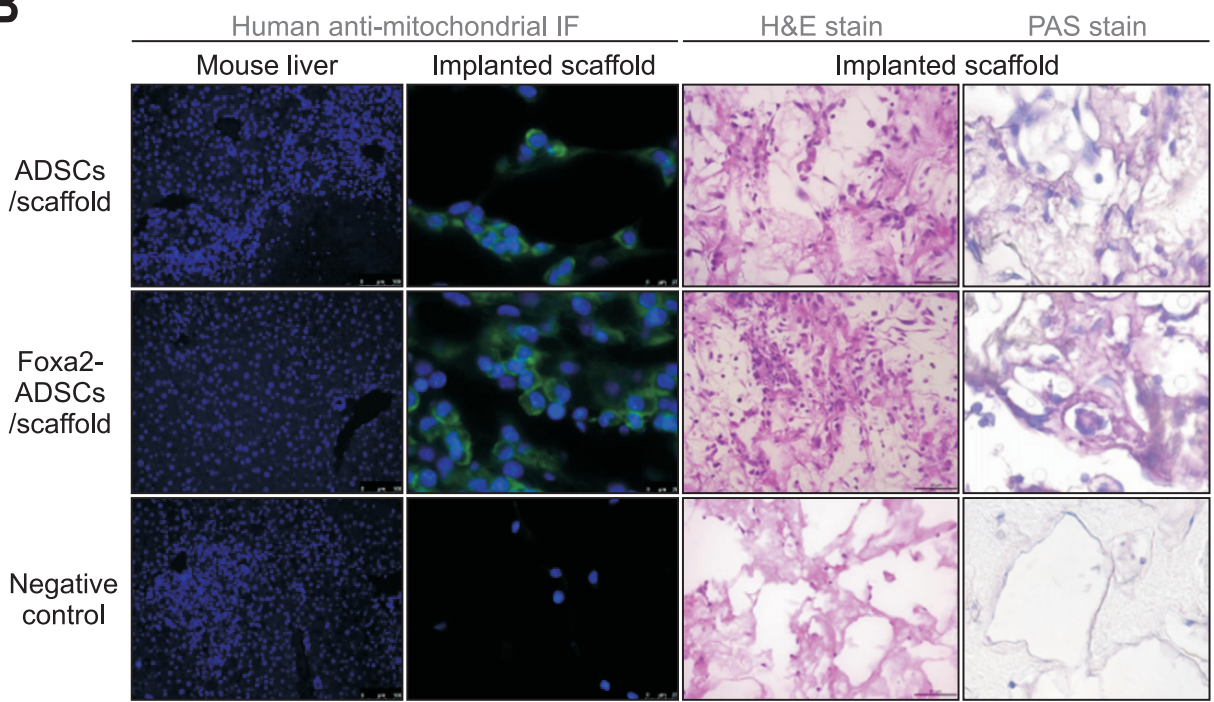

Fig. 5. Stem cell homing. (A) Mouse and human 32 -microglobulin mRNA expression was compared between the TAA-only group and the ADSCs scaffold group. (B) Immunofluorescence stain using human anti-mitochondria antibody was performed in mouse liver ( $\times 200)$ and implanted scaffold $(\times 630)$ (immunofluorescence: blue - nuclei, green - human mitochondria). H\&E staining (blue - nuclei, pink - cytosol, $\times 400)$ and periodic acid-Schiff (PAS) staining (blue - nuclei, purple - glycogen in cytoplasm, x630) of implanted scaffold.

TAA, thioacetamide; ADSCs, adipose tissue-derived stem cells; Foxa2, forkhead box protein. pressing $\mathrm{ADSC} / \mathrm{scaffold}$ group than in the non-overexpressing group (Fig. 5B).

\section{Paracrine effect of Foxa2-overexpressing ADSCs/scaf- folds}

To determine the mechanism underlying improvements in liver damage, we examined the concentrations of secreted cytokines and hormones in non-overexpressing ADSC conditional medium and Foxa2-overexpressing ADSC conditional medium. HGF and bFGF levels were higher in both ADSC treatment groups than in the control group on day 5. However, HGF, bFGF, and VEGF-A levels increased to higher levels in the Foxa2-overexpressing ADSC/scaffold group than in the simple ADSC treatment group (Fig. 6). Cytokine array data showed that the levels of both anti-inflammatory and inflammatory cytokines were higher in the ADSC groups (the Foxa2-overexpressing $\mathrm{ADSC} / \mathrm{scaffold}$ group as well as the simple ADSC group) than in the control group. IL-10, IL-4, IL-6, and IL-13 levels were also higher in the Foxa2-overexpressing ADSC/scaffold group than in the simple ADSC group and the control group. Although IL-1 $\beta$ concentrations were higher in the Foxa2overexpressing $\mathrm{ADSC} /$ scaffold group than in the simple ADSC group, TNF- $\alpha$ and MCP-1 levels did not different significantly between the groups.

\section{DISCUSSION}

The current study showed that Foxa2 gene overexpression in ADSCs promoted hepatocyte-like cell differentiation and protected hepatocytes in a liver injury model. The gene delivery system using scaffolds showed a protective effect in damaged liver tissue without stem cell migration.

Previous studies using stem cell therapy with gene transfer have reported that HNF $4 \alpha$, Foxa2, C/EBP $\alpha$ and HNF $1 \alpha$ gene overexpression could promote hepatocyte differentiation efficiency in liver diseases. ${ }^{13,18,19}$ In addition, some studies have found that Foxa2-overexpressing rBM-MSCs have enhanced function and are more effective for anti-inflammatory and antifibrotic treatment than conventional stem cell therapies. ${ }^{7}$ However, no study has evaluated the protective effects of enhanced ADSC differentiation into hepatocyte-like cells or the effects of enhanced ADSC differentiation on liver tissue using a liver disease model. Moreover, only a few studies have evaluated whether the enhanced function of Foxa2-overexpressing rBMMSCs can aid in restoring liver function or reducing damage in liver injury animal models.

In the current study, the Foxa2 gene was selected to enhance stem cell function. Both Foxa2 and GATA4 overexpression increased the expression of AFP, ALB and CK18 mRNA as well as the amount of glycogen in the cytoplasm. However, GATA4 overexpression in ADSCs reduced the viability of the cells. Therefore, in the current study, Foxa2 overexpression was selected for further experiments.

This current study demonstrates, for the first time, how stem cell function can be enhanced using gene overexpression and a scaffold system. The efficacy and safety of improving stem cell function with a scaffold system were confirmed in a mouse model of acute liver failure. A scaffold is a 3D culture system for 

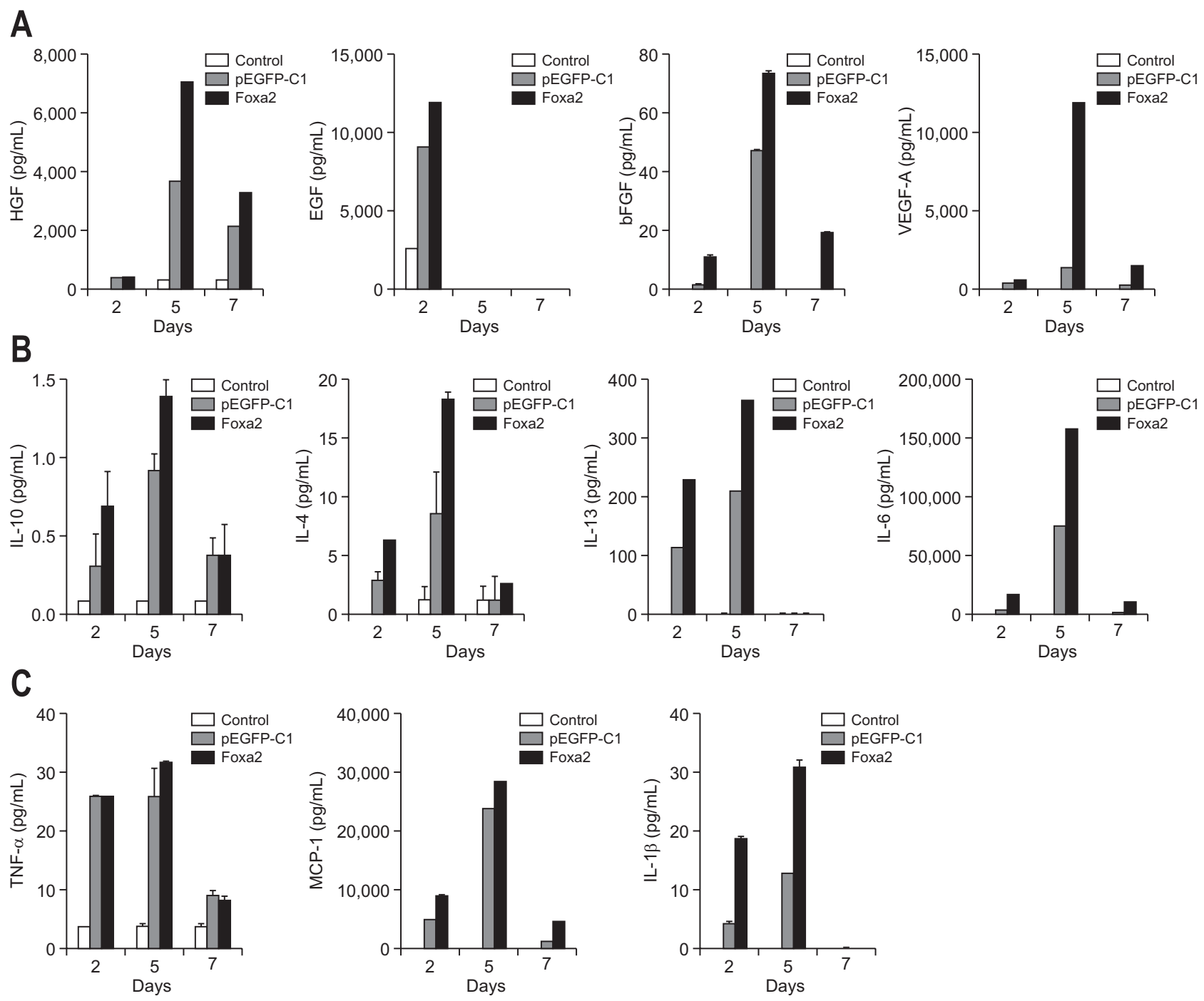

Fig. 6. Paracrine effects of cytokines. Growth factors and inflammatory cytokines were evaluated using a magnetic Luminex assay kit. On the 2nd day, differentiation medium \#1 was used. On the 5th and 7th days, differentiation medium \#2 was used as a control treatment. (A) Growth factors. (B) Anti-inflammatory cytokines. (C) Pro-inflammatory cytokines. Foxa2-overexpressing adipose tissue-derived stem cells (ADSCs) expressed various cytokines relative to ADSCs on day 5.

HGF, hepatocyte growth factor; Foxa2, forkhead box protein; EGF, epidermal growth factor; bFGF, basic fibroblast growth factor; VEGF-A, vascular endothelial growth factor-A; IL-6, interleukin 6; TNF- $\alpha$, tumor necrosis factor- $\alpha$; MCP1, monocyte chemotactic protein-1.

reproducing cell-to-cell and cell-to-environment interactions. Scaffolds are known to inhibit free migration of stem cells and to aid in stem cell proliferation and differentiation. Scaffolds generally control pore size to allow the entry of nutrients and various factors but prevent cell migration after cell attachment. As the stem cells are located on all sides of the scaffolds, unlike in 2D culture, the scaffold system provides an environment similar to that of the body to promote efficient proliferation and differentiation. ${ }^{20,21}$

One previous study treated liver diseases using scaffolds by forming 3D cultures and promoting differentiation efficiency, while another study enhanced stem cell function using hepatocyte cocultures. ${ }^{22,23}$ Therefore, we aimed to increase stem cell stability and differentiation efficiency using nonviral methods and scaffolds without stem cell homing in a process that can be applied clinically. Our study highlights the potential of using an individual's own ADSCs, which can be programmed to differentiate into hepatocyte-like cells. Moreover, the use of a nonviral system can also improve safety in humans, and using scaffolds can improve survival. All these techniques can be used in humans and can help limit liver injury.

Our research objective was to analyze the potential value of partially differentiating stem cells using Foxa2 overexpression in a scaffold system to maximize paracrine effects. In general, not only Foxa2 but also GATA4 and HNF4A are needed to fully differentiate stem cells into hepatocyte-like cells. Therefore, we partially induced differentiation using single Foxa2 gene transfer, culturing cells in differentiation medium for 1 day. We also 
investigated the additional benefits of partially differentiating hepatocyte-like cells under a scaffold system.

One week later, new blood vessels entering the scaffolds were observed visually, and red blood cells were also observed in the scaffolds after staining with several reagents. Accordingly, it is presumed that various substances secreted from the cells in the scaffolds implanted into subcutaneous fat caused systemic reactions through blood vessels. AST and ALT are very well-known biomarkers of liver inflammation; however, AST and ALT levels did not decrease in our model. Although these biomarkers are commonly evaluated, they do not always accurately reflect the degree of inflammation. Histological assessment of tissue damage is the most reliable method of evaluation, and histological evaluation in the current study suggested that the necrotic area was decreased in the Foxa2-overexpressing ADSC/scaffold group compared to the non-overexpressing group (Fig. 4B). Therefore, we have clarified an anti-inflammatory effect of stem cells. In addition, the levels of proinflammatory cytokines such as MCP1 and IL-6 were decreased in the Foxa2-overexpressing ADSC/scaffold group (Fig. 4D).

We later evaluated and compared cytokine secretion between the Foxa2-overexpressing ADSC group and the ADSC group after 14 days using a cytokine array. The results suggested that there was no significant difference between the ADSC group and the Foxa2-overexpressing ADSC group (Supplementary Fig. 2, Supplementary Tables 3 and 4). However, we further found that acute liver injury was attenuated after 1 week in the Foxa2-overexpressing ADSC/scaffold group (Fig 4). Therefore, we evaluated the expression pattern of cytokines in each group before the end of the first week. The results suggested that growth factors and anti-inflammatory cytokines were increased in the Foxa2-overexpressing ADSC group compared to the nonoverexpressing group on the 5th day of differentiation (Fig. 6A and B). Our results showed that Foxa2 promotes the differentiation of ADSCs into hepatocyte-like cells, which not only act like hepatocytes but also have increased secretion of several key growth hormones as well as anti-inflammatory cytokines (HGF, bFGF, VEGF-A, IL-10, IL-4, IL-6, and IL-13). The reason that cytokine expression was decreased in the late phase is because undifferentiated cells lost their stemness, and it is thought that cytokines are expressed during differentiation rather than in differentiated hepatocytes. In addition, it is very well known that serum ALB also has protective effects against acute liver injury. Not only the expression of various cytokines but also serum ALB levels were significantly increased in Foxa2overexpressing ADSCs compared to non-overexpressing ADSCs. Notably, buildup of endotoxins during acute liver damage leads to deterioration of liver function. It has already been clinically reported that systemic endotoxin levels are increased by acute liver injury and that ALB can bind to endotoxins. Additionally, it has been reported that ALB dialysis can alleviate liver damage and increase survival rates. ${ }^{24,25}$ We have accordingly shown that acute liver injury was alleviated by several cytokines and ALB secreted in the Foxa2-overexpressing ADSC/scaffold group.

In conclusion, the use of Foxa2-overexpressing ADSCs in a scaffold system demonstrated paracrine signaling-mediated protective effects without stem cell homing.

\section{CONFLICTS OF INTEREST}

No potential conflict of interest relevant to this article was reported.

\section{ACKNOWLEDGEMENTS}

This work was supported by a grant from SK Chemical Research Fund of the Korean Society of Gastroenterology in 2013 and the National Research Foundation of Korea (NRF), funded by the Korean Government (NRF-2016R1A6A3A11932549). The funding source had no role in study design, implementation, data collection, analysis, and interpretation, or in the preparation, review, or approval of the manuscript.

\section{AUTHOR CONTRIBUTIONS}

D.W.J. contributed to study design and grant; J.S.L., H.T.K., W.K.S. did animal care and analyzed the data; J.H.L. made scaffold; Y.J.C. wrote the manuscript; K.J. interpreted histologic finding.

\section{ORCID}

Yeon Ji Chae https://orcid.org/0000-0001-8609-7082 Dae Won Jun https://orcid.org/0000-0002-2875-6139 Jai Sun Lee https://orcid.org/0000-0002-1673-8777 Waqar Khalid Saeed https://orcid.org/0000-0002-7888-9108 Hyeon Tae Kang https://orcid.org/0000-0002-4771-3388 Kiseok Jang https://orcid.org/0000-0002-6585-3990 Jin Ho Lee https://orcid.org/0000-0002-1528-3416

\section{REFERENCES}

1. Kern S, Eichler H, Stoeve J, Klüter H, Bieback K. Comparative analysis of mesenchymal stem cells from bone marrow, umbilical cord blood, or adipose tissue. Stem Cells 2006;24:1294-1301.

2. Prasajak P, Leeanansaksiri W. Developing a new two-step protocol to generate functional hepatocytes from Wharton's jelly-derived mesenchymal stem cells under hypoxic condition. Stem Cells Int 2013;2013:762196.

3. Lee JS, Lee JM, Im GI. Electroporation-mediated transfer of Runx2 and Osterix genes to enhance osteogenesis of adipose stem cells. Biomaterials 2011;32:760-768.

4. Lan YW, Choo KB, Chen CM, et al. Hypoxia-preconditioned mesenchymal stem cells attenuate bleomycin-induced pulmonary 
fibrosis. Stem Cell Res Ther 2015:6:97.

5. Shams S, Mohsin S, Nasir GA, Khan M, Khan SN. Mesenchymal stem cells pretreated with HGF and FGF4 can reduce liver fibrosis in mice. Stem Cells Int 2015;2015:747245.

6. Hu X, Xie P, Li W, Li Z, Shan H. Direct induction of hepatocytelike cells from immortalized human bone marrow mesenchymal stem cells by overexpression of HNF4alpha. Biochem Biophys Res Commun 2016;478:791-797.

7. Cho JW, Lee CY, Ko Y. Therapeutic potential of mesenchymal stem cells overexpressing human forkhead box A2 gene in the regeneration of damaged liver tissues. J Gastroenterol Hepatol 2012;27:1362-1370.

8. Kim MD, Kim SS, Cha HY, et al. Therapeutic effect of hepatocyte growth factor-secreting mesenchymal stem cells in a rat model of liver fibrosis. Exp Mol Med 2014;46:e110.

9. Tang Y, Li Q, Meng F, et al. Therapeutic potential of HGF-expressing human umbilical cord mesenchymal stem cells in mice with acute liver failure. Int J Hepatol 2016;2016:5452487.

10. Walesky C, Apte U. Role of hepatocyte nuclear factor 4alpha (HNF4alpha) in cell proliferation and cancer. Gene Expr 2015;16:101108.

11. Ishii K, Yoshida Y, Akechi Y, et al. Hepatic differentiation of human bone marrow-derived mesenchymal stem cells by tetracycline-regulated hepatocyte nuclear factor 3beta. Hepatology 2008;48:597-606.

12. Yamamoto Y, Teratani T, Yamamoto H, et al. Recapitulation of in vivo gene expression during hepatic differentiation from murine embryonic stem cells. Hepatology 2005;42:558-567.

13. Ding Y, Chang C, Niu Z, et al. Overexpression of transcription factor Foxa2 and Hnf1alpha induced rat bone mesenchymal stem cells into hepatocytes. Cytotechnology 2016;68:2037-2047.

14. Jung Y, Bauer G, Nolta JA. Concise review: induced pluripotent stem cell-derived mesenchymal stem cells: progress toward safe clinical products. Stem Cells 2012;30:42-47.

15. Furlani D, Ugurlucan M, Ong L, et al. Is the intravascular administration of mesenchymal stem cells safe? Mesenchymal stem cells and intravital microscopy. Microvasc Res 2009;77:370-376.

16. Gnecchi M, Zhang Z, Ni A, Dzau VJ. Paracrine mechanisms in adult stem cell signaling and therapy. Circ Res 2008 21;103:12041219.

17. Tang YL, Zhao Q, Qin X, et al. Paracrine action enhances the effects of autologous mesenchymal stem cell transplantation on vascular regeneration in rat model of myocardial infarction. Ann Thorac Surg 2005;80:229-236.

18. Liu T, Zhang S, Xiang D, Wang Y. Induction of hepatocytelike cells from mouse embryonic stem cells by lentivirusmediated constitutive expression of Foxa2/Hnf4a. J Cell Biochem 2013;114:2531-2541.

19. Iacob R, Rüdrich U, Rothe M, et al. Induction of a mature hepatocyte phenotype in adult liver derived progenitor cells by ectopic expression of transcription factors. Stem Cell Res 2011;6:251-261.

20. Oh SH, Park IK, Kim JM, Lee JH. In vitro and in vivo characteristics of PCL scaffolds with pore size gradient fabricated by a centrifugation method. Biomaterials 2007;28:1664-1671.

21. Oh SH, Kim TH, Jang SH, Im GI, Lee JH. Hydrophilized 3D porous scaffold for effective plasmid DNA delivery. J Biomed Mater Res A 2011;97:441-450.

22. Li J, Tao R, Wu W, et al. 3D PLGA scaffolds improve differentiation and function of bone marrow mesenchymal stem cell-derived hepatocytes. Stem Cells Dev 2010;19:1427-1436.

23. Carrao A, Buggio M, Gardin C, Tedeschi U, Ferroni L, Zavan PB. Mesenchymal stem cells increase neo-angiogenesis and albumin production in a liver tissue-engineered engraftment. Int J Mol Sci 2016;17:374.

24. Lee KC, Baker LA, Stanzani G, et al. Extracorporeal liver assist device to exchange albumin and remove endotoxin in acute liver failure: results of a pivotal pre-clinical study. J Hepatol 2015;63:634-642.

25. Karvellas CJ, Gibney N, Kutsogiannis D, Wendon J, Bain VG. Bench-to-bedside review: current evidence for extracorporeal albumin dialysis systems in liver failure. Crit Care 2007;11:215. 